\title{
Translational cooling and storage of protonated proteins in an ion trap at subkelvin temperatures
}

\author{
D. Offenberg, C. B. Zhang, Ch. Wellers, B. Roth, and S. Schiller \\ Institut für Experimentalphysik, Heinrich-Heine-Universität Düsseldorf, 40225 Düsseldorf, Germany
}

\begin{abstract}
Gas-phase multiply charged proteins have been sympathetically cooled to translational temperatures below $1 \mathrm{~K}$ by Coulomb interaction with laser-cooled barium ions in a linear ion trap. In one case, an ensemble of 53 cytochrome c molecules (mass $\simeq 12390 \mathrm{amu}$, charge $+17 e$ ) was cooled by $\simeq 160$ laser-cooled barium ions to less than $0.75 \mathrm{~K}$. Storage times of more than 20 minutes have been observed and could easily be extended to more than an hour. The technique is applicable to a wide variety of complex molecules.
\end{abstract}

In the last years, the development of techniques for producing cold molecules $(<1 \mathrm{~K})$ has moved into focus. Remarkable advances in this young field of quantum physics are enabling studies of light-molecule, atom-molecule and molecule-molecule interactions in a temperature regime not accessible previously. Cold molecule types produced so far range from neutral diatomic molecules produced by photoassociation in ultracold atomic gases [1] to polar few-atom molecules slowed down by Stark deceleration [2] and charged diatomic to polyatomic molecules sympathetically cooled by lasercooled atomic ions in ion traps $[\underline{3},[4,[5]$. The heaviest cold molecular species prepared had a mass of $410 \mathrm{amu}$.

It is of interest to develop methods that allow a similar control over much heavier particles like anorganic clusters or biomolecules, consisting of hundreds or thousands of atoms. So far, such complex systems have been studied in ion traps using buffer gas cooling [6, 7]. The buffer gas thermalizes with a cryogenically cooled chamber containing the trap and collisionally cools the trapped ions internally and translationally to temperatures of $\sim 10 \mathrm{~K}$ [8]. Recently we have reported on a technique for cooling polyatomic molecular ions that allows one to achieve significantly lower translational temperatures and long storage times of up to hours. As examples, singly protonated dye (mass $410 \mathrm{amu}$ ) [5] and glycyrrethinic acid molecules (mass $471 \mathrm{amu}$ ) 9] produced by electrospray ionization (ESI) have been sympathetically cooled to less than $150 \mathrm{mK}$ by their mutual Coulomb interaction with laser-cooled ${ }^{138} \mathrm{Ba}^{+}$ions in a linear quadrupole trap.

Here we demonstrate a major extension of this technique to much heavier molecules (such as proteins), taking full advantage of the ability of the ESI ion source to produce multiply charged ions with mass-to-charge ratios suitable for conventional quadrupole mass filters and linear ion traps [10, 11]. We have produced, trapped, sympathetically cooled, and maintained cold for extended intervals 12 - and 17 -fold protonated protein molecules (cytochrome c, a protein consisting of about 100 amino acids with a total mass of $\simeq 12390 \mathrm{amu}$ ) using Doppler lasercooled ${ }^{138} \mathrm{Ba}^{+}$ions in a linear quadrupole trap. The two trapped species were detected and distinguished via excitation of their specific motional resonances. The sym- pathetic cooling was qualitatively proven by measuring the energy distribution of the ions and quantitatively analyzed using molecular dynamics simulations.

Our apparatus [5] is shown schematically in Fig. 1. It consists of an ESI ion source for the production of singly and multiply protonated molecular ions, a quadrupole mass filter for the selection of specific molecular species, a rf octopole ion guide to transfer the selected molecular ions from the medium vacuum region of the apparatus to an ultrahigh vacuum (UHV) chamber, and a linear quadrupole trap in this UHV chamber to store the molecular ions for cooling and further investigations. The preparation of the laser-cooled ${ }^{138} \mathrm{Ba}^{+}$ion ensembles used for sympathetic cooling of the molecular ions as well as the required lasers (493 and $650 \mathrm{~nm}$ ) is described in [4]. With sufficient cooling power the barium ions arrange in ordered structures, so-called Coulomb crystals, that can

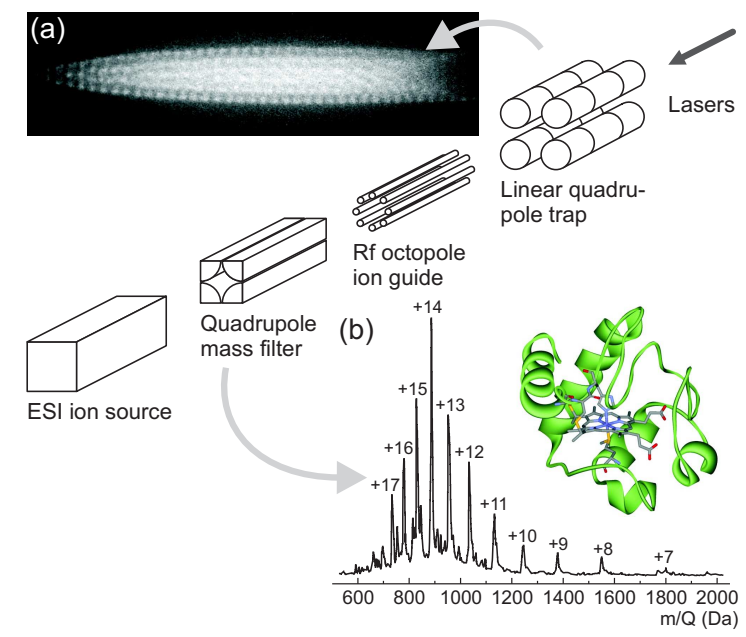

FIG. 1: (Color online) Schematic overview of our setup. Singly or multiply protonated molecular ions from an ESI ion source are selected by a quadrupole mass filter and transferred via a RF octopole ion guide to a linear quadrupole trap in an UHV chamber. (a) CCD image of a typical trapped barium ion Coulomb crystal used as coolant for the molecular ions. (b) Secondary structure and ESI mass spectrum of cytochrome c showing the distribution of the different grades of protonation. 
be imaged with a charge-coupled-device (CCD) camera [Fig. 1 (a)]. The images provide information not only on the fluorescing ${ }^{138} \mathrm{Ba}^{+}$ions, but indirectly also on the numbers and temperatures of the invisible sympathetically cooled molecular ions. Molecular dynamics (MD) simulations are used to derive these data from structural deformations and sharpness of the barium ion Coulomb crystals [12].

Multiply protonated molecules of cytochrome c from horse heart (Fluka BioChemika), (cyt c $+n \mathrm{H})^{n+}$ with grades of protonation, $n=7-17$, have been produced by ESI from a solution concentration of $10^{-5} \mathrm{M}$ in $1: 1$ methanol:water. As the maximum $n$ depends on the $p \mathrm{H}$ value of the solution, a solution with a higher concentration of acetic acid of $2 \%$ was used to produce 17 -fold protonated cytochrome c molecules, here denoted as $\mathrm{Cyt}^{17+}$, and a lower concentration of $0.5 \%$ was used to obtain 12fold protonated molecules $\mathrm{Cyt}^{12+}$.

The preparation procedures for sympathetically cooled $\mathrm{Cyt}^{12+}$ and $\mathrm{Cyt}^{17+}$ ion ensembles are identical. First, helium buffer gas at room temperature is injected into the trap chamber to pressures of $1-10 \times 10^{-5}$ mbar in order to subsequently reduce the kinetic energy of the molecular ions from the octopole ion guide so far that they can be trapped. After loading ions of the desired molecular species for $1-10 \mathrm{~s}$ depending on the desired ion number and the ion flux (typically several thousand ions per second reach the trap), the buffer gas is pumped away and the background pressure in the trap chamber returns to $<1 \times 10^{-9}$ mbar within a few minutes. Then, barium ions from an evaporator oven are loaded into the trap and cooled by the two cooling laser beams propagating along the trap axis. Impurity ions generated during loading (such as molecular fragments or $\mathrm{CO}_{2}^{+}$from the evaporator) can be removed from the trap by applying an additional appropriately strong ac voltage to the trap electrodes in order to excite the ions' specific motional resonances beyond stability [13]. At a typical rf amplitude of $450 \mathrm{~V}$ the radial resonance frequency of ${ }^{138} \mathrm{Ba}^{+}$ions is $118 \mathrm{kHz}$, while that of cytochrome ions is in the range of $10-22 \mathrm{kHz}$ depending on their protonation grade. At the end of the preparation procedure, two frequency scans $(125-1000$ and $110-40 \mathrm{kHz})$ are applied to purify the barium-cytochrome ion ensemble.

The same principle of radial excitation can be used in a nondestructive way to identify trapped ion species. For this purpose, frequency scans with $\sim 10$ times lower amplitudes than for purification are performed while acquiring the barium ions' fluorescence intensity with a photomultiplier. Under resonance excitation of a particular ion species, its motion heats all trapped ions due to their mutual Coulomb interaction. As a consequence, the barium ions' fluorescence drops, leading to dips in the radial excitation spectrum at the species-specific resonance frequencies [15, 16]. $\mathrm{Cyt}^{12+}$ and $\mathrm{Cyt}^{17+}$ in barium-ion Coulomb crystals have been identified and distinguished

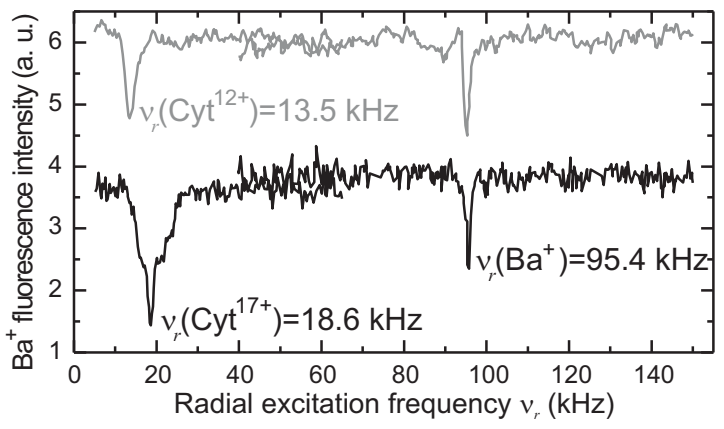

FIG. 2: Radial excitation spectra of barium crystals containing differently protonated cytochrome ions. The upper $\left(\mathrm{Cyt}^{12+}\right)$ and lower $\left(\mathrm{Cyt}^{17+}\right)$ curves were acquired at the same trap parameters and yield the same barium frequency of $95.4 \mathrm{kHz}$ used for calibration. The obtained cytochrome frequencies are well confirmed by their theoretical values.

via their radial resonance frequencies as shown in Fig. 2. The upper and lower curves are composed of two different scans: one from 5 to $65 \mathrm{kHz}$ for the detection of the molecular ions and one from 40 to $150 \mathrm{kHz}$ with a 10 times lower amplitude for the barium ions (in order to avoid crystal destruction). As the rf amplitude was the same for all four scans $(362 \mathrm{~V})$, the barium resonances coincide at $\nu_{r}\left(\mathrm{Ba}^{+}\right)=95.4 \mathrm{kHz}$ and can be used for calibration. With the resonance frequency $\nu_{r} \sim Q / m$ (for $\left.-a \ll q[13]\right)$ the expected frequencies for (noninteracting) cytochrome ions can be calculated as $\nu_{r}\left(\mathrm{Cyt}^{n+}\right)=\nu_{r}\left(\mathrm{Ba}^{+}\right) \cdot\left[Q\left(\mathrm{Cyt}^{n+}\right) / Q\left(\mathrm{Ba}^{+}\right)\right]$. $\left[m\left(\mathrm{Ba}^{+}\right) / m\left(\mathrm{Cyt}^{n+}\right)\right]$, which yields $12.8 \mathrm{kHz}$ for $\mathrm{Cyt}^{12+}$ and $18.1 \mathrm{kHz}$ for $\mathrm{Cyt}^{17+}$. These values are well confirmed by the experimental results of 13.5 and $18.6 \mathrm{kHz}$. The small differences of less than $5 \%$ can be ascribed to various factors like the direction and amplitude of the frequency scans [15] or motional coupling effects [16].

Additionally, we have implemented a destructive mass identification technique which is based on a massselective extraction of the ions from the trap. When decreasing the trap rf amplitude $U_{\text {rf }}$ the trapping stability parameter $q$ [13] is reduced and ions escape the trap at a mass-to-charge ratio dependent amplitude $U_{\mathrm{rf}}^{\mathrm{ex}} \propto \sqrt{m / Q}$ 17. In our setup an ion detector below the trap registers the escaping ions during such a controlled reduction of the trap rf amplitude, leading to an ion extraction mass spectrum as shown in Fig. 3. Although this technique is destructive and less accurate than the radial excitation, it has several advantages. It is fast (an extraction takes a few seconds) and it works also with uncooled ions. Moreover, it provides qualitative information on the ion temperatures as the mass spectra of warmer ion ensembles are broadened due to the ions' wider energy distribution. This is shown in Fig. 3 for $\mathrm{Ba}^{+} / \mathrm{Cyt}^{17+}$ ensembles in the cooled (black) and the noncooled case (gray). In the cooled case, both the peak of the laser-cooled $\mathrm{Ba}^{+}$ions and the peak of the $\mathrm{Cyt}^{17+}$ ions are narrower. This is a 


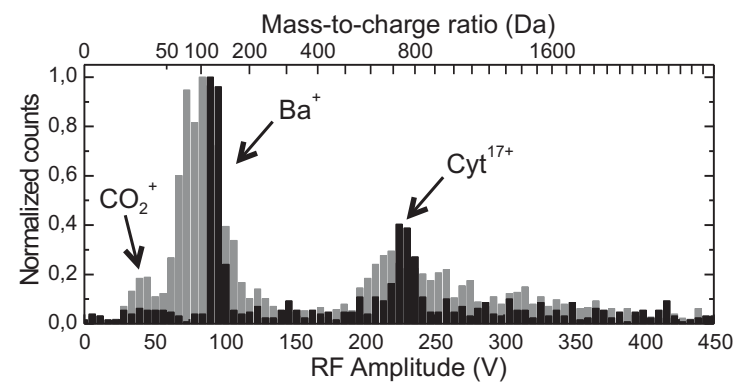

FIG. 3: Ion extraction mass spectra of $\mathrm{Ba}^{+} / \mathrm{Cyt}^{17+}$ ensembles at different temperatures. In the noncooled case (gray) the mass spectrum is broadened compared to the laser-cooled case (black). Here, not only the peak of the laser-cooled $\mathrm{Ba}^{+}$ions, but also that of the simultaneously trapped $\mathrm{Cyt}^{17+}$ ions is narrower, which is a proof of their sympathetic cooling.

qualitative proof of their sympathetic cooling.

$\mathrm{Ba}^{+} / \mathrm{Cyt}^{n+}$ crystals were routinely produced. Several $\mathrm{Ba}^{+} / \mathrm{Cyt}^{12+}$ and $\mathrm{Ba}^{+} / \mathrm{Cyt}^{17+}$ ion crystals have been analyzed quantitatively by the use of MD simulations in order to obtain the numbers of the trapped ions and their translational temperatures $T_{\mathrm{sec}, i}=\frac{2}{3}\left\langle E_{i}\right\rangle / k_{B}$, where $\left\langle E_{i}\right\rangle$ is the time- and subensemble-averaged secular kinetic energy per ion of the species $i$. Our MD method is described in [5] and [12]. Here, we do not consider micromotion in the simulations explicitly since it does not affect the CCD images [18]. Micromotion-related effects, such as rf heating, are taken into account phenomenologically.

In a quadrupole trap ions of different charge-to-mass ratios arrange radially separated according to their effective radial trap potentials $\Phi(r) \propto \frac{Q}{m} r^{2}[13$ ] and the interspecies repulsion $\sim Q_{1} Q_{2}$. In the limit of cylindrical symmetry, this radial separation scales as $r_{1} / r_{2}=$ $\left(Q_{2} m_{1} / Q_{1} m_{2}\right)^{1 / 2}$ with the outer radius $r_{1}$ of the lower mass-to-charge ratio $\left(m_{1} / Q_{1}\right)$ subensemble and the inner radius $r_{2}$ of the higher mass-to-charge ratio $\left(m_{2} / Q_{2}\right)$ subensemble [19]. As shown in Figs. 目(a) and 团(d), the multiply charged cytochrome ions form a sheath around the barium ions which radially squeezes and axially prolongs the barium ion subensemble. In the experiment two CCD images of the ion crystals are recorded: one with the molecular ions present and one after the molecular ions have been removed. In the case presented in Figs. 4 (a)4 (c) the $\mathrm{Cyt}^{17+}$ ions prolong the barium ion subensemble axially by $240 \mu \mathrm{m}$ [Fig. (4) (b) and 4(c)], which can be explained by the presence of $53 \mathrm{Cyt}^{17+}$ ions, according to MD simulations. The barium ensemble consists of 158 laser-cooled ${ }^{138} \mathrm{Ba}^{+}$ions and 230 barium isotopes other than ${ }^{138} \mathrm{Ba}$. Due to nonoptimal trap parameters during the experiment of Fig. 4 (b), the observed radial squeezing of the barium ion subensemble is less pronounced than in the simulated image.

For the temperature determination a specific heating rate $h_{i}=d\left\langle E_{i}\right\rangle / d t$ for each ion species $i$ is chosen that phenomenologically describes heating effects such as col-
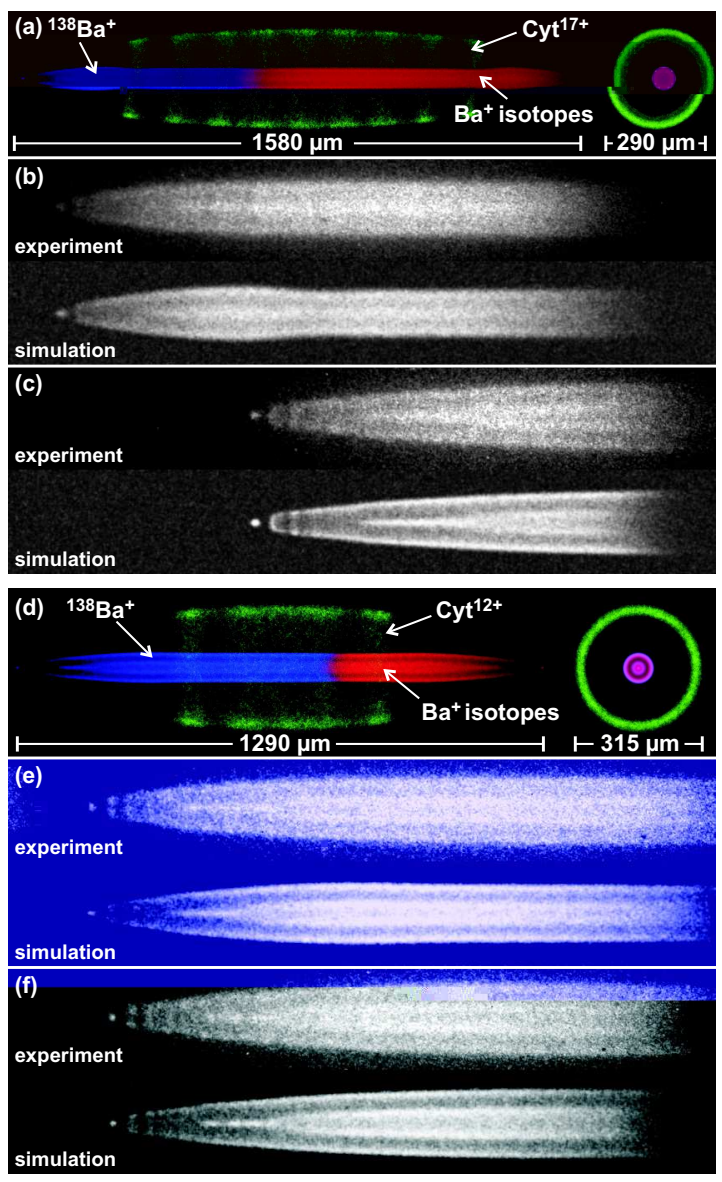

FIG. 4: (color online) Experimental and simulated CCD images of $\mathrm{a} \mathrm{Ba}^{+} / \mathrm{Cyt}^{17+}$ and $\mathrm{a} \mathrm{Ba}^{+} / \mathrm{Cyt}^{12+}$ ion crystal. (a, d) Simulated images in radial (left) and axial view (right), as they would appear if all ions would fluoresce. The cooling lasers propagate to the left, separating the ${ }^{138} \mathrm{Ba}^{+}$ions (blue) and the barium isotopes (red) axially due to the light pressure force. The cytochrome ions (green) form a sheath around the barium ion subensemble. (b, e) Experimental image of the barium-cytochrome ion crystal and its simulation, showing only the fluorescing ${ }^{138} \mathrm{Ba}^{+}$ions. (c, f) Crystals after the removal of the cytochrome ions. Here, the barium ion ensemble is no longer deformed nor heated by the molecular ions.

lisions with background gas particles, rf heating, or electric field noise [12]. For the laser-cooled ${ }^{138} \mathrm{Ba}^{+}$ions a cooling rate is employed corresponding to the laser cooling force $F_{D}=-\beta v_{z}$, where $\beta$ is the damping coefficient and $v_{z}$ the $z$ component of the ion velocity with the cooling lasers propagating in the positive $z$ direction [5]. The other species are cooled sympathetically through the interparticle Coulomb interaction, resulting in a steady state in which every ion species $i$ has its specific translational temperature $T_{\mathrm{sec}, i}$. For the pure $\mathrm{Ba}^{+}$-ion crystal shown in Fig. (4) (c) a heating rate $h_{\mathrm{Ba}^{+}}=\frac{3}{2} k_{B}(3.7 \mathrm{~K} / \mathrm{s})$ [20] needs to be applied to reproduce the experimental CCD image concerning shape and internal structure of the ${ }^{138} \mathrm{Ba}^{+}$ion subensemble. Here, a 
realistic damping coefficient $\beta=1.7 \times 10^{-22} \mathrm{~kg} / \mathrm{s}$ was assumed [21], which yields the temperatures $T_{\mathrm{sec},{ }^{138} \mathrm{Ba}^{+}}=$ $19 \mathrm{mK}$ and $T_{\text {sec, } \mathrm{Ba}^{+} \text {isotopes }}=23 \mathrm{mK}$. As all conditions remained constant, the same damping coefficient $\beta$ and the same heating rate $h_{\mathrm{Ba}^{+}}$are applied to reproduce the CCD image of the corresponding "parent" $\mathrm{Ba}^{+} / \mathrm{Cyt}^{17+}$ ion crystal [Fig. 4(b)]. Here, a heating rate $h_{\mathrm{Cyt}^{17+}}=\frac{3}{2} k_{B}(71.6 \mathrm{~K} / \mathrm{s})$ must be present in order to reproduce the observed warmer barium ion subensemble whose temperatures are $T_{\text {sec, }{ }^{138} \mathrm{Ba}^{+}}=66 \mathrm{mK}$ and $T_{\text {sec,Bat }}{ }^{\text {isotopes }}=109 \mathrm{mK}$. With this value, the cytochrome temperature is $T_{\mathrm{sec}, \mathrm{Cyt}^{17+}}=704 \mathrm{mK}$. As the laser cooling efficiency varies depending on numerous factors, we consider a range of $\beta$ values, as observed by 22]. To obtain a lower (upper) limit for the heating rates and temperatures we simulate the cases $\beta=1.0(2.0) \times$ $10^{-22} \mathrm{~kg} / \mathrm{s}$. For the ion crystal shown in Fig. 4(b) with $T_{\mathrm{sec},{ }^{138} \mathrm{Ba}^{+}}=66 \mathrm{mK}$, this yields the following values: $h_{\mathrm{Ba}^{+}}=\frac{3}{2} k_{B}\left(3.7_{-1.6}^{+0.6} \mathrm{~K} / \mathrm{s}\right), T_{\mathrm{sec}, \mathrm{Ba}}{ }^{+}$isotopes $=109_{-17}^{+6} \mathrm{mK}$, $h_{\mathrm{Cyt}^{17+}}=\frac{3}{2} k_{B}\left(71.6_{-29.2}^{+11.9} \mathrm{~K} / \mathrm{s}\right)$, and $T_{\mathrm{sec}, \mathrm{Cyt}^{17+}}=$ $704_{-24}^{+26} \mathrm{mK}$.

The temperature of the molecular ions mainly depends on the laser cooling power, the pressure in the vacuum chamber, and the ratio of the numbers of laser-cooled and sympathetically cooled ions and also their mass-to-charge ratio compared to that of the laser-cooled ${ }^{138} \mathrm{Ba}^{+}$ions. The closer the ratios are, the lower is the radial separation of the two species in the trap and the higher is the sympathetic cooling efficiency. In the cases shown in Fig. 4, the radial gap $\left(r_{2}-r_{1}\right)$ between the $\mathrm{Cyt}^{17+}$ and the ${ }^{138} \mathrm{Ba}^{+}$ion ensembles is $64 \mu \mathrm{m}$ and in the $\mathrm{Cyt}^{12+}$ case $87 \mu \mathrm{m}$. This contributes to the fact that the $\mathrm{Cyt}^{12+}$ ions could only be cooled to $1.2 \mathrm{~K}$, although the barium ions at $26 \mathrm{mK}$ were much colder than in the $\mathrm{Cyt}^{17+}$ case [23]. As a consequence of the different cytochrome temperatures, the $\mathrm{Cyt}^{12+}$ subensemble [Fig. 4(d)] appears more blurred in the simulated images than the colder

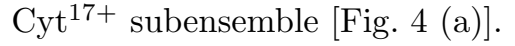

In conclusion, we have shown that heavy and thus complex particles, appropriately charged, can be translationally cooled in the gas phase to temperatures significantly below those reachable with other techniques. The lowest temperature observed was below $0.75 \mathrm{~K}$. The temperature is limited by heating experienced by the molecular ions. Its origin is uncertain so far, but may be due to electrical noise. Combining the present result with earlier work (sympathetic cooling of masses up to $410 \mathrm{amu}$ ) [4, 5] we reasonably expect that ions of any mass between 32 and 13000 amu can be sympathetically cooled, provided they have an adequately high charge. The particular advantage of this cooling method is that the ions can be stored for more than 20 min (easily extendable to hours using an improved vacuum to avoid loss reactions of the ${ }^{138} \mathrm{Ba}^{+}$ions) with a strong spatial localization in a well-defined and nearly collisionless environment. These are ideal conditions for, e. g., studies of low-energy processes, like cold collisions or triplet-singlet decay, or highresolution spectroscopy for structural or conformational characterizations of biomolecules. For many experiments cooling of the internal degrees of freedom of the molecules would be favorable and could be implemented by radiative cooling in a cryogenic environment.

We thank T. Schneider for helpful discussions. D.O. acknowledges support from the Studienstiftung des deutschen Volkes and C.B.Z. from the Deutscher Akademischer Austauschdienst (DAAD).

[1] A. Fioretti et al., Phys. Rev. Lett. 80, 4402 (1998).

[2] F. M. H. Crompvoets et al., Nature (London) 411, 174 (2001).

[3] M. Drewsen, A. Mortensen, R. Martinussen, P. Staanum, and J. L. Sørensen, Phys. Rev. Lett 93, 243201 (2004).

[4] B. Roth et al., J. Phys. B 38, 3673 (2005).

[5] A. Ostendorf et al., Phys. Rev. Lett. 97, 243005 (2006); 100, 019904(E) (2008).

[6] D. Gerlich et al., Planet. Space Sci 50, 1275 (2002).

[7] O. Asvany et al., Science 309, 1219 (2005).

[8] J. A. Stearns et al., J. Am. Chem. Soc. 129, 13820 (2007).

[9] D. Offenberg et al. (to appear in J. Phys. B).

[10] J. B. Fenn et al., Science 246, 64 (1989).

[11] S. Schiller and C. Lämmerzahl, Phys. Rev. A 68, 053406 (2003).

[12] C. B. Zhang, D. Offenberg, B. Roth, M. A. Wilson, and S. Schiller, Phys. Rev. A 76, 012719 (2007).

[13] A linear quadruple trap produces an effective electric potential $\Phi(r, z)=\frac{1}{2} \frac{m}{Q} \omega_{r}^{2} r^{2}+\frac{1}{2} \frac{m}{Q} \omega_{z}^{2} z^{2}$ with the ion's massto-charge ratio $\frac{m}{Q}$; the radial distance from the trap axis, $r$; and the axial distance from the trap center, $z$. In this potential an ion can radially and axially oscillate with specific frequencies given by $\omega_{r}=\frac{\Omega}{2} \sqrt{q^{2} / 2+a}$ and $\omega_{z}=\frac{\Omega}{2} \sqrt{-2 a}$. The parameters $q$ and $a$ determine the trapping stability [14] and are given by $q=\frac{2 Q U_{\mathrm{rf}}}{m r_{0}^{2} \Omega^{2}}$ and $a=\frac{-4 \kappa Q U_{E C}}{m \Omega^{2}}$. Typical trap parameters in our case are $U_{\mathrm{rf}}=200-500 \mathrm{~V}$ for the rf amplitude, $U_{\mathrm{EC}}=5-7 \mathrm{~V}$ for the dc potential difference between end and middle electrodes; constant parameters are the rf frequency $\Omega=2 \pi \times 2.5 \mathrm{MHz}$, the axis-to-electrode-surface distance $r_{0}=4.36 \mathrm{~mm}$, and the geometrical factor $\kappa=1500 \mathrm{~m}^{-2}$.

[14] M. Drewsen and A. Brøner, Phys. Rev. A 62, 045401 (2000).

[15] T. Baba and I. Waki, J. Appl. Phys. 92, 4109 (2002).

[16] B. Roth, P. Blythe, and S. Schiller, Phys. Rev. A 75, 023402 (2007).

[17] The relationship $U_{\mathrm{rf}}^{\mathrm{ex}} \propto \sqrt{m / Q}$ was derived from an analysis of the stability diagram [14] with regard to the decrease of $q$ below stability when reducing $U_{\text {rf }}$. It holds when the edge of the stability range is approximated by $q=-\frac{1}{2} a^{2}$ (adequate for the range of $q$ considered here).

[18] Here, the micromotion energy [19] is $25 \mathrm{meV}$ for $\mathrm{Cyt}^{12+}$ and $38 \mathrm{meV}$ for $\mathrm{Cyt}^{17+}$. However, for spectroscopy experiments with laser beams parallel to the trap axis this purely radial micromotion should not be relevant. 
[19] L. Hornekær, Ph.D. thesis, Aarhus University, 2000.

[20] Due to their low mass difference, the heating rates for the ${ }^{138} \mathrm{Ba}^{+}$and the barium isotope ions are assumed equal.

[21] Ch. Raab et al., Phys. Rev. Lett. 85, 538 (2000).

[22] P. Bushev et al., Phys. Rev. Lett. 92, 223602 (2004).

[23] For the $\mathrm{Ba}^{+} / \mathrm{Cyt}^{12+}$ ion crystal shown in Fig. 4 (d) the simulations yield $170{ }^{138} \mathrm{Ba}^{+}$ions at $26 \mathrm{mK}$ and 280 barium isotopes at $35_{-5}^{+2} \mathrm{mK}$ with $h_{\mathrm{Ba}}{ }^{+}=\frac{3}{2} k_{B}\left(4.8_{-1.9}^{+1.6} \mathrm{~K} / \mathrm{s}\right)$, and $28 \mathrm{Cyt}^{12+}$ ions at $1.2_{-0.28}^{+0.05} \mathrm{~K}$ with $h_{\mathrm{Cyt}^{12+}}=$ $\frac{3}{2} k_{B}\left(60.1_{-25.0}^{+7.9} \mathrm{~K} / \mathrm{s}\right)$. 